OPEN ACCESS

Edited by:

Huaxi Yi,

Ocean University of China, China

Reviewed by:

Huizi Tan,

Nanchang University, China

Jufang Wang,

South China University of Technology,

China

${ }^{*}$ Correspondence:

Minqi Wang

wangmq@zju.edu.cn

Specialty section:

This article was submitted to

Nutritional Immunology,

a section of the journal

Frontiers in Immunology

Received: 13 August 2021 Accepted: 08 November 2021 Published: 30 November 2021

Citation:

Xiao Z, Liu L, Pei X, Sun W, Jin Y, Yang S-T and Wang $M$ (2021) A Potential Probiotic for Diarrhea: Clostridium tyrobutyricum Protects Against LPS-Induced Epithelial

Dysfunction via IL-22 Produced

By Th17 Cells in the lleum.

Front. Immunol. 12:758227. doi: 10.3389/fimmu.2021.758227

\section{A Potential Probiotic for Diarrhea: Clostridium tyrobutyricum Protects Against LPS-Induced Epithelial Dysfunction via IL-22 Produced By Th17 Cells in the lleum}

\author{
Zhiping Xiao ${ }^{1}$, Lujie Liu ${ }^{1}$, Xun Pei ${ }^{1}$, Wanjing Sun ${ }^{1}$, Yuyue Jin ${ }^{1}$, Shang-Tian Yang ${ }^{2}$ \\ and Minqi Wang ${ }^{1 *}$ \\ 1 The key Laboratory of Molecular Animal Nutrition, Ministry of Education, College of Animal Sciences, Zhejiang University, \\ Hangzhou, China, ${ }^{2}$ Department of Chemical and Biomolecular Engineering, The Ohio State University, Columbus, \\ $\mathrm{OH}$, United States
}

Probiotics are clinically used for diarrhea and inflammatory bowel diseases in both humans and animals. Previous studies have shown that Clostridium tyrobutyricum (Ct) protects against intestinal dysfunction, while its regulatory function in the gut needs further investigation and the related mechanisms are still not fully elucidated. This study aims to further verify the protective function of $\mathrm{Ct}$ and reveal its underlying mechanisms in alleviating diarrhea and intestinal inflammation. Ct inhibited LPS-induced diarrhea and intestinal inflammation in the ileum. IL-22 was identified and the protective role of $\mathrm{Ct}$ in the ileum presented an IL-22-dependent manner according to the transcriptomic analysis and in vivo interference mice experiments. The flow cytometric analysis of immune cells in the ileum showed that Ct enhanced the proportions of Th17 cells in response to LPS. The results of in situ hybridization further verified that Ct triggered Th17 cells to produce IL-22, which combined with IL-22RA1 expressed in the epithelial cells. Moreover, Ct was unable to enhance the levels of short-chain fatty acids (SCFAs) in the ileum, suggesting that the protective role of $\mathrm{Ct}$ in the ileum was independent of SCFAs. This study uncovered the role of $\mathrm{Ct}$ in alleviating diarrhea and inflammation with the mechanism of stimulating Th17 cells in the lamina propria to produce IL-22, highlighting its potential application as a probiotic for diarrhea and inflammation in the ileum.

Keywords: Clostridium tyrobutyricum ${ }_{1}$, diarrhea ${ }_{2}$, intestinal barrier function ${ }_{3}$, IL-22 $_{4}$, Th17 cells 5

\section{INTRODUCTION}

Diarrhea is a gastrointestinal condition with high morbidity and mortality. Generally, diarrhea results from the decreased absorption of $\mathrm{Cl}^{-}$and $\mathrm{Na}^{+}$and movement of water, leading to the imbalance of ions and solute across the gut epithelium (1). When diarrhea occurs, the enteric pathogens easily disseminate into the intestinal microenvironment, disturbing homeostasis of the 
epithelial barrier, host flora, and immune cells, inducing intestinal inflammation, causing intestinal inflammation, and gastrointestinal diseases like inflammatory bowel disease (IBD).

Alleviation of diarrhea and intestinal inflammation requires an ingenious intestinal barrier system concerting the crosstalk between the microbiota and immune cells to avoid excessive immune responses to commensal microbes or pathogens. This process involves a series of mechanisms, including the secretion of cytokines, chemokines, and immunomodulatory molecules, such as thymic stromal lymphopoietin, TGF- $\beta$, retinoic acid, IL10, and IL-5 produced by intestinal epithelial cells (IECs) (2). The mechanisms involved in microbiota-IECs-immune cell interactions are still being elucidated because of the cell culture systems imitating the physiological cues of the complex gastrointestinal mucosa microenvironment. However, equilibrium of the gut microbiota, IECs, and immune cells is crucial for intestinal homeostasis.

Clostridium tyrobutyricum (C. tyrobutyricum/Ct) is one of the anaerobic Clostridium strains. Compared with other gram-positive bacteria, the cell well of $C$. tyrobutyricum is mainly made up of higher protein (35-40\% dry weight), lower insoluble peptidoglycan (10-12\% dry weight), and neutral sugar, and absent teichuronic acids $(3,4)$. Numerous studies have revealed that Clostridium tyrobutyricum could be used to produce higher butyric acid via bacterial fermentation (5-7). Recently, C. tyrobutyricum has been revealed to protect barrier function in different tissues, including the gut (8), testis (9), and endometrium (10). A new study about the genomic analysis of $C$. tyrobutyricum pointed out that C. tyrobutyricum is a potential bacteria in regulating health in human beings (11). Our earlier study has shown that C. tyrobutyricum protects against lipopolysaccharide (LPS)induced epithelial dysfunction in IPEC-J2 cells (12). Moreover, C. tyrobutyricum has been revealed in alleviating LPS-induced inflammation via regulating immune cells in the intestinal sections $(13,14)$. However, the regulatory function of C. tyrobutyricum in the gut needs further elucidation and its underlying mechanism in maintaining intestinal homeostasis is still not fully understood.

In this study, we aimed to investigate the role and possible mechanisms of C. tyrobutyricum in the ileum based on our previous study (14). We further proved that C. tyrobutyricum prevented diarrhea and protected against LPS-induced ileal barrier dysfunction in vivo. IL-22 was identified according to the transcriptomic analysis of the ileum. C. tyrobutyricum alleviated LPS-induced epithelial destruction depending on IL-22 produced according to the in vivo interference experiments. We also revealed that C. tyrobutyricum enhanced the MHC-II process, thereby stimulating Th17 cells to produce IL-22 which combined with IL-22RA1 expressed in the epithelial cells. Strikingly, $C$. tyrobutyricum decreased the levels of short-chain fatty acids (SCFAs) in response to LPS, suggesting that the regulatory function of $C$. tyrobutyricum in the gut was independent of SCFAs. Our studies revealed the role of $C$. tyrobutyricum in the crosstalk between epithelial cells and immune cells mediated by IL-22 and identifies the potential application of C. tyrobutyricum as a probiotic for diarrhea and inflammation in the ileum.

\section{MATERIALS AND METHODS}

\section{Antibodies and Reagents}

LPS (derived from Escherichia coli strain O55:B5) and Collagenase VIII from Clostridium histolyticum (C2139) were from SigmaAldrich (USA). The antibodies in the flow cytometry were described as our previous studies $(13,14)$. All antibodies used in the flow cytometric analysis, immunoblotting, and immunohistochemistry were shown in Supplemental Table 1. Transcription Factor Staining Buffer Set (562574) and Stain Buffer FBS (554656) were from BD (USA). HEPES was from Gibco (USA). EDTA and BacLight ${ }^{\text {TM }}$ Green Bacterial Stain (B-35000) were from Invitrogen (USA). Fetal Bovine Serum was purchased from Gemini (USA). RMPI 1640 was from BI (Israel). TRIzol reagent, The PrimeScript RT reagent kit with gDNA Erase, and SYBR Green qPCR reagent were from Takara (Japan). DNase (D8070), 5\% BSA (SW3015), Triton X-100 (P1080) and DAPI (C0065) were from Solarbio (China). The Opal 7-color Manual IHC kit (NEL811001KT) was purchased from PerkinElmer (USA). Polybrene was provided by HanBio (China). The whole cell lysis assay (KGP2100) was from KeyGEN (China).

\section{Mice}

Four-week-old C57BL/6 male mice were purchased from Shanghai SLAC Laboratory Animal Co., Ltd and bred at the Zhejiang University Laboratory Animal Center $\left(25^{\circ} \mathrm{C}, 12 / 12-\mathrm{h}\right.$ light/dark cycle) allowing unrestricted access to standard mice diet and water. The mice experiments were performed following the protocol approved by the Institutional Animal Care and Use Committee of Zhejiang University. For the mice feeding trial, four-week C57BL/6 male mice were treated with PBS (Control, mice were treated with PBS by gavage for 20 days followed by intraperitoneal injection with $\mathrm{PBS}, \mathrm{n}=10$ ), $\mathrm{Ct}$ (mice were treated with $10^{8} \mathrm{CFU} / \mathrm{mL} \mathrm{Ct}$ for 20 days followed by intraperitoneal injection with PBS, $n=10$ ), LPS (mice were treated with PBS for 20 days followed by intraperitoneal injection with $10 \mathrm{mg} / \mathrm{kg}$ BW LPS, $\mathrm{n}=12$ ), and Ct + LPS (mice were treated with $10^{8} \mathrm{CFU} / \mathrm{mL} \mathrm{Ct}$ for 20 days followed by intraperitoneal injection with $10 \mathrm{mg} / \mathrm{kg} \mathrm{BW}$ LPS, $\mathrm{n}=12$ ). For the in vivo interference feeding experiment, adeno-associated virus 2/2-m-shIL22 labeled with GFP (AAVshIL22) and AAV-shNC were generated by Hanbio (Shanghai, China). The sequences of AAV-shNC and AAV-shIL22 were designed as the previous study, namely 5'-TTCTCCGAAC GTGTCACGTAA-3' and 5'-GCTAAGGATCAGTGCTACCTG ATGA-3' (13). Four-week C56BL/6 male mice were first intraperitoneally injected with $200 \mu \mathrm{L}$ AAV-shIL22/AAVshILNC at a concentration of at least $2.4 \times 10^{11} \mathrm{vg} / \mathrm{mL}$. After 14 days, mice were treated with PBS (AAV-shNC-Control, $n=19$; AAV-shIL22-Control, n=17), Ct (AAV-shNC-Ct, n=14, AAVshIL22-Ct, $\mathrm{n}=12$ ), LPS (AAV-shNC-LPS, n=20; AAV-shIL22LPS, $\mathrm{n}=18$ ), and Ct + LPS (AAV-shNC-Ct + LPS, $\mathrm{n}=17$; AAV-shIL22-Ct + LPS, $n=12$ ), respectively as described above.

\section{Bacterial Culturing}

C. tyrobutyricum was provided by Prof. Shang-Tian Yang, from The Ohio State University and was cultured as our previous 
study (5). Simply, C. tyrobutyricum was cultured anaerobically at $37^{\circ} \mathrm{C}$ in a clostridial growth medium (CGM), collected after centrifugation at 12,000 rpm for $5 \mathrm{~min}$, and suspended in PBS.

\section{Bacterial Colonization}

For the colonization of C. tyrobutyricum in the intestine, $10^{8}$ $\mathrm{CFU} / \mathrm{mL}$ C. tyrobutyricum was first mixed with $100 \mu \mathrm{M}$ working solution of the BacLight bacterial stain, incubated for $15 \mathrm{~min}$ at room temperature, and washed with PBS. C57BL/6 mice were then treated with $C$. tyrobutyricum by gavage and the intestinal samples were collected, frozen with liquid nitrogen, embedded with OCT, and sectioned. The fluorescence was visualized using a confocal microscope LSM 880 and captured using ZEN 2.3 software (Carl Zeiss, Germany).

\section{RNA-Sequencing}

Total RNA of ileal samples from the Control, LPS, Ct, and Ct + LPS groups $(n=6)$ were isolated for RNA-Sequencing. RNA sequencing was performed by Novogene using an Illumina sequencer. Clean reads obtained by removing reads containing adapter, poly- $\mathrm{N}$ and low-quality reads were aligned to the reference genome using Tophat2 RNA-Seq alignment software. HTSeq was used to count the read numbers mapped to each gene. Differential expression analysis was conducted using the DESeq $\mathrm{R}$ package. The $\mathrm{P}$ values were adjusted using the Benjamini \& Hochberg method (FDR). Corrected P-value of 0.05 and fold-change of at 1 was set as the threshold for significantly differential expression.

\section{Immune Cells Isolation and Flow Cytometric Analysis}

The isolation of immune cells from the ileal lamina propria was performed as previously described $(15,16)$. The steps of cell isolation and antibodies staining were conducted according to our previous studies $(13,14)$. Simply, the ileal sections were isolated from the mice, washed with PBS, cut into $5-\mathrm{cm}$ pieces after removing the fat tissue, mesenteric, and Peyer's patches, and shaken in PBS containing 2\% FBS, $10 \mathrm{mM}$ HEPES, and $2 \mathrm{mM}$ EDTA at $37^{\circ} \mathrm{C}$ for $30 \mathrm{~min}$ for two to three cycles. The intestinal pieces were then shaken in RPMI 1640 medium containing 10\% FBS, $2 \mathrm{mM}$ L-glutamine, $100 \mathrm{U} / \mathrm{mL}$ penicillin, $100 \mu \mathrm{g} / \mathrm{mL}$ streptomycin, $0.6 \mathrm{mg} / \mathrm{mL}$ collagenase VIII, and $150 \mu \mathrm{g} / \mathrm{mL}$ DNase at $37^{\circ} \mathrm{C}$ for $30 \mathrm{~min}$ for two cycles, passed through 100 $\mu \mathrm{m}$ and $40 \mu \mathrm{m}$ cell strainers, and centrifuged at $400 \times \mathrm{g}$ for $10 \mathrm{~min}$. Cells were then stained with Live/Dead (FVS780) for $20 \mathrm{~min}$ followed by CD16/32 antibody for $15 \mathrm{~min}$, washed with PBS, stained with surface antibodies for $20 \mathrm{~min}$, and washed with PBS. For the intracellular antibodies, cells were fixed and permeabilized for $2 \mathrm{~h}$ followed by staining the intracellular antibodies for $2 \mathrm{~h}$, washed with PBS, and suspended in PBS before processing. The data were analyzed with FlowJo software (BD, USA).

\section{Transmission Electron Microscopy and Scanning Electron Microscopy}

Ileal sections were washed in PBS and fixed in 2.5\% GA overnight. All samples were washed in PBS and postfixed in $1 \%$ osmic acid for 1-2 $\mathrm{h}$. Then the samples were washed in PBS and dehydrated in a series of gradient ethanol solutions (50\%, $75 \%, 85 \%, 95 \%$, and $100 \%$ ethanol), each for $15 \mathrm{~min}$. For SEM, the samples were dehydrated in a Hitachi Model HCP-2 critical point dryer with liquid $\mathrm{CO}_{2}$ and visualized using a Philips Model SU8010 FASEM (Hitachi, Japan). For TEM, samples were embedded in Epon resin and cut into $60 \mathrm{~nm}$ ultrathin sections. Sections were counterstained with uranyl acetate and lead citrate. All the samples were observed using a Hitachi HT7650 electron microscope (Hitachi, Japan).

\section{Histology and Immunohistochemistry}

Ileal samples were fixed overnight in 4\% PFA, and then dehydrated in $30 \%$ sucrose in PBS solution for $48 \mathrm{~h}$ until sunk to the bottom. Ileal sectioning, $\mathrm{H} \& \mathrm{E}$, and $\mathrm{ZO}-1$ staining were performed by Zhejiang Chinese Medical University. The scanning was conducted on Nikon Eclipse 80i (Nikon, Japan). The intestinal morphology was evaluated with NDP. View2 (Hamamatsu, China). The average of density (AOD) of ZO-1 was obtained with ImageJ software.

\section{In situ Hybridization}

For tissue fluorescence in situ hybridization, the Opal 7-color Manual IHC kit was used according to the manual provided. The sections were dewaxed with xylene, rehydrated through a graded series of ethanol solutions, and fixed in 10\% neutral buffered formalin. After microwave treatment and blocking, the ileal sections were sequentially stained with primary antibodies and HRP-conjugated secondary antibodies. One of four Opal reagents was used for staining followed by microwave treatments and another round of staining. Slides were finally stained with DAPI for $5 \mathrm{~min}$ and mounted before image acquisition. CD45, EpCAM, CD3e, ROR $\gamma$ t, IL-22, and IL22RA1 were used as primary antibodies. The dyes Opal 520, Opal540, Opal570, and Opal 590 were used for staining. Images were visualized using confocal microscope LSM 880 and captured using ZEN 2.3 software (Carl Zeiss, Germany). Considering the autofluorescence of tissues, the contrast/ brightness with consistent parameters was regulated in all images using ZEN 2.3 software. All images were analyzed with Image J software.

\section{RT-qPCR}

Total RNA was extracted using TRIzol reagent. cDNA was synthesized with PrimeScript RT reagent kit with gDNA Eraser according to the manufacturer's instructions. RT-qPCR was performed on the CFX96TM Real-Time System (Bio-Rad) in duplicate or triplicate. Data were analyzed according to the 2 $\triangle \triangle \mathrm{Ct}$ method and normalized to the expression of GAPDH. PCR primers are shown in Supplemental Table 2.

\section{Immunoblotting}

Whole-cell lysates of ileum was prepared using Whole Cell Lysis Assay. After electrophoresis with 10\% SDS-PAGE, proteins were transferred to PVDF membranes. The membranes were blocked with $5 \%$ non-fat milk for $1 \mathrm{~h}$ at room temperature, incubated with specific primary antibodies followed by HRP-conjugated secondary antibodies, and detected by the ECL reagent. 


\section{Gas Chromatographic Analysis}

The concentrations of SCFAs were analysed via gas chromatography $(17,18)$ The ileal contents were mixed with 5 $\mathrm{mL}$ double distilled water for $1 \mathrm{~h}$. The supernatant was collected after centrifugation at $10,000 \mathrm{~g}$ for $15 \mathrm{~min}$ and mixed with $85 \%$ orthophosphoric acid for $1 \mathrm{~h}$. The supernatant was then collected, passed by $0.22 \mu \mathrm{m}$ strainers, and transferred into the gas chromatography vial. The concentrations of SCFAs in the ileal contents of mice were measured by GC-8A gas chromatography (Shimadzu, Kyoto, Japan).

\section{Statistical Analysis}

All statistical tests were performed with Prism 8.0 software and analyzed using two-tail unpaired t-test. All data are expressed as mean \pm SEM and $P<0.05$ was considered significant and the level of significance was indicated as ${ }^{\star} P<0.05,{ }^{*} P<0.01$.

\section{RESULTS}

\section{Ct Alleviates LPS-Induced Ileal Barrier Dysfunction}

Our previous studies have investigated the most efficient concentration of $\mathrm{Ct}$ in alleviating intestinal dysfunction and suggested that $10^{8} \mathrm{CFU} / \mathrm{mL} \mathrm{Ct}$ effectively alleviated LPSinduced inflammation in different sections of the intestine including the duodenum, ileum, and colon $(13,14)$. To further understand the role of $\mathrm{Ct}$ in alleviating diarrhea and intestinal inflammation in vivo, we first evaluated the colonization of $\mathrm{Ct}$ in vivo via staining $\mathrm{Ct}$ with BacLight ${ }^{\mathrm{TM}}$ green. Mice were treated with $10^{8} \mathrm{CFU} / \mathrm{mL} \mathrm{Ct}$ by gavage. After $4 \mathrm{~h}$, faint fluorescence was observed on the lumen and the villus surface of the small intestines (Supplemental Figure 1), indicating that Ct could colonize in the small intestines, while its colonization capacity in the intestine was weak.

Mice were then treated with $10^{8} \mathrm{CFU} / \mathrm{mL} \mathrm{Ct}$ by gavage followed by LPS injection. Compared with LPS group, Ct maintained the body weight gain (Figure 1A) and inhibited diarrhea (Figure 1B) in response to LPS. In response to LPS, Ct improved the ileal morphology, enhanced the villus height, and villus height/crypt depth ratio (Figure 1C), which were consistent with our previous study (14). Compared with LPS group, Ct significantly increased the expression of ZO-1 (a typical tight junction protein) in the ileum in response to LPS (Figure 1D). Under electron microscopy, we observed that LPS induced the destruction of villi, microvilli, and tight junction structures, while Ct improved these phenomena in response to LPS (Figures 1E, F). These results further verified that $\mathrm{Ct}$ effectively inhibited diarrhea and alleviated ileal barrier dysfunction induced by LPS.

\section{Ct Enhances the Expression of IL-22 in the lleum}

The ileal samples were collected after the feeding experiment and transcriptomic analysis was conducted to elucidate the molecular mechanism of $\mathrm{Ct}$ in the ileum. A total of 19 differentially expressed genes were identified between the $\mathrm{Ct}$ and Control groups according to padj $<0.05$ and $\mid \log _{2}$ (FoldChange) $\mid>1$ (Figure 2A and Supplemental Table 3). Ct up-regulated genes like Ahsg and Alb, which have been revealed in inhibiting intestinal diseases such as ulcerative colitis $(19,20)$. In addition, Ct down-regulated genes including Tacr2, Cav1, Adrb3, Csf3, and Cxcl2, which enriched in 'Neuroactive ligand-receptor interaction', 'Calcium signaling pathway', 'Endocytosis', and 'Cytokine-cytokine receptor' processes (Supplemental Figure 2A and Supplemental Table 4). These results indicated that $\mathrm{Ct}$ inhibited intestinal inflammation and regulated intestinal nervous response. A total of 145 differentially expressed genes were identified between the LPS and Ct + LPS groups (Figure 2B, Supplemental Table 5). Compared with the LPS group, genes including Maf, IL-18, H2Ab1, H2-DMa, H2-Eb1, Gsdmd, Ahsg, Alb, and Gc enriching in 'Intestinal bowel disease' and bacterial infection were enhanced in the Ct + LPS group. In addition, Ct regulated the intestinal metabolism, especially fatty acid metabolism in response to LPS (Supplemental Figure 2B and Supplemental Table 6). Altogether, we suggested three possible mechanisms of $\mathrm{Ct}$ in regulating intestinal immune response, namely neuro-, metabolism-, and cytokines-immune regulation. In this study, we focused on the mechanism of cytokines-immune regulation.

The mRNA expression of Csf3, Gsdmd, and Maf was first determined. No differences in the mRNA expression of Csf3, Gsdmd, and Maf were observed between the LPS and Ct + LPS groups (Figure 2C), which were inconsistent with the transcriptomic analysis. In the innate immune system, pathogens/ extracellular stimuli are recognized by pattern-recognition receptors (PRRs) like Toll-like receptors (TLRs) and NOD-like receptors (NLRs) to induce the recruitment of monocytes, thereby producing cytokines and chemokines $(21,22)$. According to the transcriptomic analysis, we also noticed that Ct decreased the expression of TLRs such as TLR4, a well-known receptor of LPS (Supplemental Figure 3A, Supplemental Table 7). Clostridium butyricum, another Clostridium strain, which has been clinically used in regulating intestinal health with the mechanism via activating TLR2/MyD88 signaling pathway in colitis (23). Unlike Clostridium butyricum, Ct decreased the expression of TLR2 in response to LPS, indicating that Ct regulated intestinal health in a TLR2-independent manner. NLRP9b is highly expressed in the ileal epithelial cells and restricts rotavirus infection (24). In this study, Ct decreased the mRNA expression of NLRP6 and NLRP9b in response to LPS (Supplemental Figure 3B).

To address whether $\mathrm{Ct}$ regulated intestinal immune response via cytokines. Herein, some differentially expressed cytokines and chemokines were identified among the Control, Ct, LPS, and Ct + LPS groups (Figure 2D and Supplemental Tables 8, 9). We then analyzed the mRNA expression of these cytokines and chemokines in the ileum using RT-qPCR. Ct decreased the mRNA expression of IL-1 $\beta$, IL-6, TNF- $\alpha, \mathrm{Ccl} 2, \mathrm{Ccl} 9$, and Ccl11 in response to LPS (Figure 2E and Supplemental Figure 3D). Besides, in response to LPS, Ct decreased the levels of IL- 6 , IL- $1 \beta$, TNF- $\alpha$, and IFN- $\gamma$ in the ileum according to ELISA analysis (Figure 2F). The above results showed that $\mathrm{Ct}$ inhibited LPS-induced inflammation in the ileum. We also 


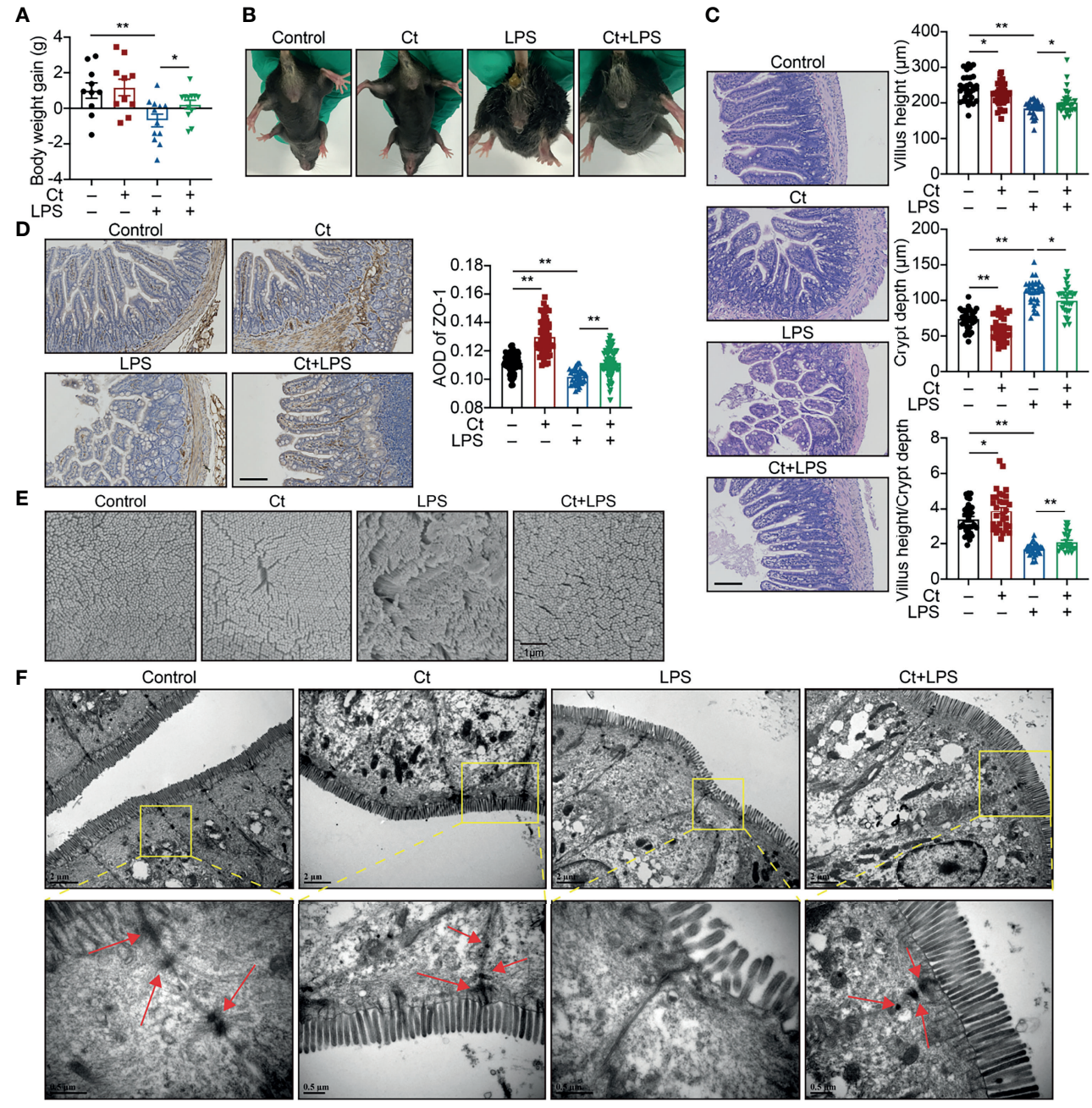

FIGURE 1 | Ct alleviates LPS-induced diarrhea and ileal barrier function in vivo. C57BL/6 were randomly divided into 4 groups, including the Control (n=10), Ct $(n=10)$, LPS ( $n=12)$, Ct + LPS ( $n=12)$. All samples were collected after 24 h. (A) Body weight gain. (B) Diarrhea. (C, D) H\&E staining (C) and ZO-1 expression (D). The presented figures of H\&E and ZO-1 staining in each group were from the same sample. Scale bar: 500 um. (E) Visualization of villus morphology under SEM. Scale bar: $1 \mu \mathrm{m}$. (F) Visualization of microvillus and structure of tight junctions under TEM. Scale bar: $2 \mu \mathrm{m}$. Data were presented as mean \pm SEM. The significant difference was analyzed by two-way unpaired t-tests. ${ }^{\star} P<0.05,{ }^{\star \star} P<0.01$.

noticed that Ct enhanced the mRNA expression of IL-18, IL$17 \mathrm{rc}$, and IL-22 in response to LPS (Figure 2E). Some earlier studies have shown that these three cytokines (IL-18, IL-17, and IL-22) induce intestinal inflammation (25-27), while recent studies show the protective roles of these cytokines in the gut (28-30). These guided us to hypothesize that Ct might regulate intestinal inflammation via enhancing IL-18, IL-17, and IL-22 in the ileum.

According to the results of RNA-seq and RT-qPCR verification, in response to LPS, Ct enhanced the mRNA expression of H2-Eb1 and H2-DMa in the ileum (Figure 2G), which have been defined as major histocompatibility complex class II-related transcripts (MHC-II) (31). Generally, MHC-II is constitutively expressed on antigen-presenting cells and epithelial cells, recognizing the antigen derived from nutrients, commensal bacteria, or pathogens, and is sensitized by $\mathrm{CD} 4^{+} \mathrm{T}$ cells, thereby promoting immune response (32-35). MHC-II deficiency accounts for higher susceptibility to enteric infections, leading to intestinal inflammation and gastrointestinal disease like IBD (36). We then analyzed the proportions of $\mathrm{MHC}-\mathrm{II}^{+}$ cells in the ileum. Compared with LPS group, Ct significantly enhanced the proportion of $\mathrm{MHC}-\mathrm{II}^{+}$cells in response to LPS (Figure 2H). It has been reported that MHC-II, IL-22, and type 3 innate lymphoid cells (ILC3s) partially overlap in the intestine according to the sing-cell RNA-sequencing (37), which guided us to assume that $\mathrm{Ct}$ might regulate intestinal immune response via IL-22. We then measured the protein expression of IL-22 in the ileum and found that $\mathrm{Ct}$ enhanced the IL-22 expression in response to LPS (Figure 2I). The level of IL-22 in the ileum was also measured using ELISA. In response to LPS, Ct enhanced the level of IL-22 in the ileum (Figure 2J). Altogether, Ct might alleviate LPS-induced inflammation via IL-22 in the ileum. 


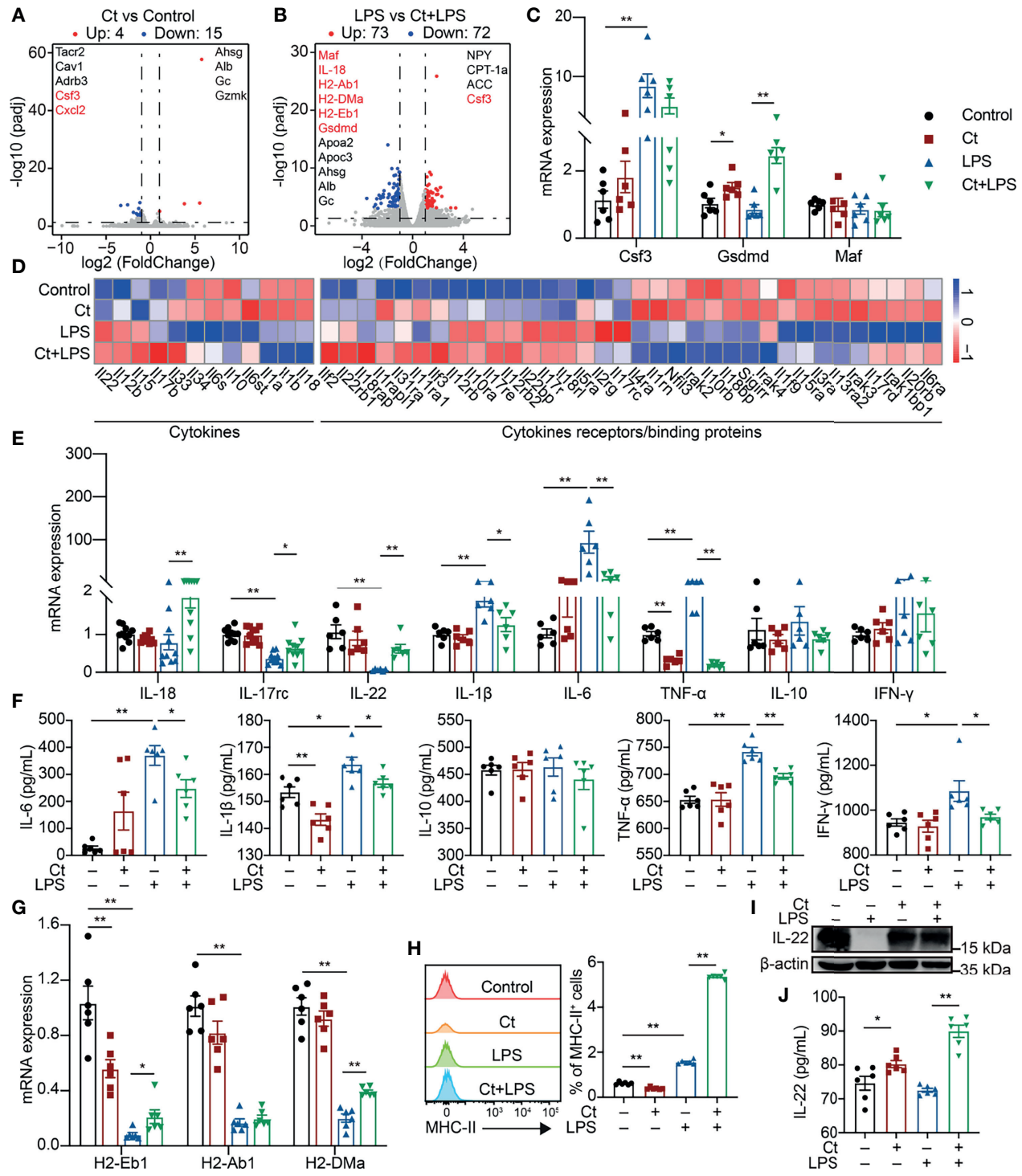

FIGURE 2 | Ct enhances the expression of IL-22 in response to LPS. (A) Volcano plots of differentially expressed genes between the Control and Ct group (Ct vs Control, n=6). (B) Volcano plots of differentially expressed genes between the LPS and Ct + LPS group (LPS vs Ct + LPS, n=6). (C) RT-qPCR analysis of Csf3, Gsdmd, and Maf in the ileum ( $n=6)$. (D) Heat-map of differentially expressed genes enriched in cytokines and cytokine receptors/binding proteins. (E) RT-qPCR analysis of cytokines including IL-18, IL-17rc, IL-22, IL-1 $\beta$, IL-6, TNF- $\alpha$, IL-10, and IFN- $\gamma$ in the ileum (IL-18 and IL-17rc, $n=10$, others, $n=6)$. (F) The levels of inflammatory cytokines including IL-6, IL-1 $\beta$, IL-10, TNF- $\alpha$, and IFN- $\gamma$ in the ileum ( $n=6)$. (G) RT-qPCR analysis of MHC- $I I$ transcripts in the ileum ( $n=6$ ). (H) Proportions of $\mathrm{MHC}-I^{+}$cell in the ileum $(\mathrm{n}=6)$. (I) Immunoblotting analysis of IL-22 in the ileum. (J) ELISA analysis of IL-22 levels in the ileum ( $\left.\mathrm{n}=6\right)$. Data were presented as mean \pm SEM. The significant difference was analyzed by two-way unpaired t-tests. ${ }^{\star} P<0.05,{ }^{\star \star} P<0.01$.

\section{Ct Protects LPS-Induced Inflammation Depending on IL-22 in the lleum}

To investigate whether $\mathrm{Ct}$ protected intestinal barrier function and alleviated LPS-induced intestinal inflammation depending on IL-22. An RNA interference experiment in vivo with adenoassociated virus-shIL22 labeled with GFP (AAV-shIL22) by intraperitoneal injection was conducted to knock down IL-22 in the intestine. The fluorescence was observed on the surface of both ileum and colon, offering the possibility for AAV-shIL22 to target the intestine (Supplemental Figure 4A). The mRNA expression of IL-22 in the ileum was reduced in mice treated with AAV-shIL22 and the efficiency of in vivo interference reached 64.19\% (Supplemental Figure 4B).

After knocking down IL-22, Ct was unable to maintain the body weight gain (Figure 3A), while Ct decreased the diarrhea incidence in response to LPS (Figure 3B). In mice treated with $\mathrm{AAV}$-shNC, Ct maintained the whole length of the intestine in response to LPS, while no differences were observed between LPS and Ct + LPS groups after knocking down IL-22 (Figure 3C). We also observed the above phenomenon in the colon and 


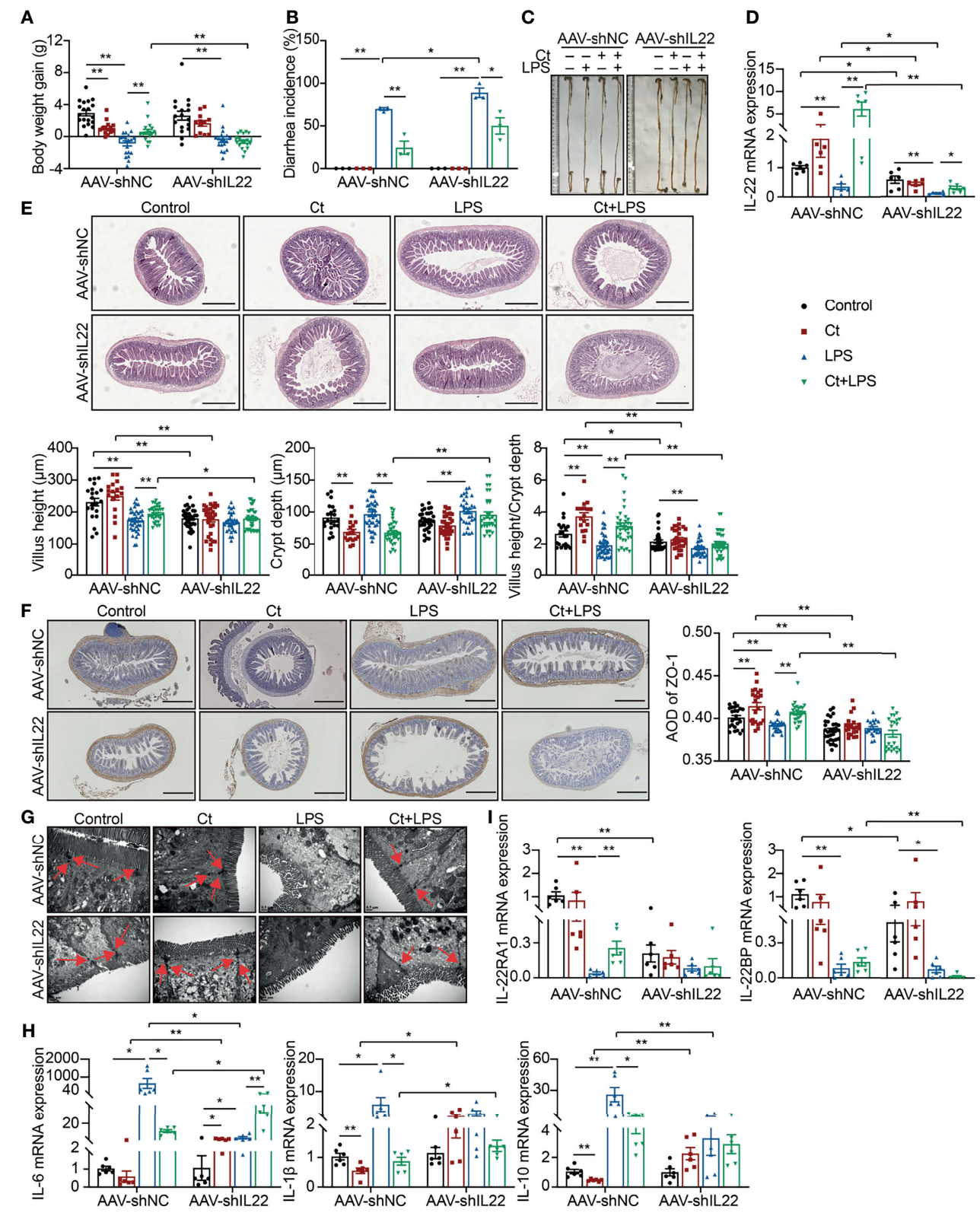

FIGURE 3 | Ct alleviates intestinal barrier dysfunction in an IL-22-dependent manner. C57BL/6 mice were first intraperitoneally injected with AAV-shNC ( $\mathrm{n}=70)$ and AAV-shlL22 ( $n=59)$, after 14 days, mice were randomly divided into 8 groups, namely AAV-shNC-Control $(n=19), A A V-s h N C-C t(n=14), A A V-s h N C-L P S(n=20), A A V$ shNC-Ct + LPS ( $n=17)$, AAV-shIL22-Control ( $n=17), A A V-s h I L 22-C t(n=12), A A V-s h I L 22-L P S(n=18), A A V-s h l L 22-C t+L P S$ ( $n=12)$. All samples were collected after 24 h. (A) Body weight gain. (B) Diarrhea incidence. (C) Intestinal length. (D) RT-qPCR analysis of IL-22 in the ileum (n=6). (E) H\&E staining. Scale bar: $500 \mu$ m. (F) ZO-1 expression. Scale bar: $500 \mu \mathrm{m}$. (G) Visualization of microvilli and structure of tight junctions under TEM. Scale bar: $0.5 \mu \mathrm{m}$. (H) RT-qPCR analysis of inflammatory cytokines including IL-6, IL-1 $\beta$, and IL-10 in the ileum ( $n=6)$. (I) RT-qPCR analysis of IL-22 and IL-22BP in the ileum ( $n=6)$. Data were presented as mean \pm SEM. The significant difference was analyzed by two-way unpaired t-tests. ${ }^{\star} P<0.05,{ }^{* \star} P<0.01$.

demonstrated that Ct alleviated colonic dysfunction in an IL-22dependent manner (13). After knocking down IL-22, compared with LPS group, Ct still enhanced the mRNA expression of IL-22 in the ileum in response to LPS (Figure 3D). Fragmentary villi were observed after knocking down IL-22 in the ileum, and in response to LPS, Ct dramatically decreased the villus height and villus height/crypt depth ratio after knocking down IL-22 compared with that in AAV-shNC-Ct + LPS group. After knocking down IL-22, Compared with LPS group, Ct was unable to enhance the villus height and villus height/crypt depth ratio in response to LPS (Figure 3E).

Compared with mice treated with AAV-shNC, the expression of ZO-1 in the Control, Ct, and Ct + LPS groups was decreased after knocking down IL-22, and Ct was unable to maintain ZO-1 


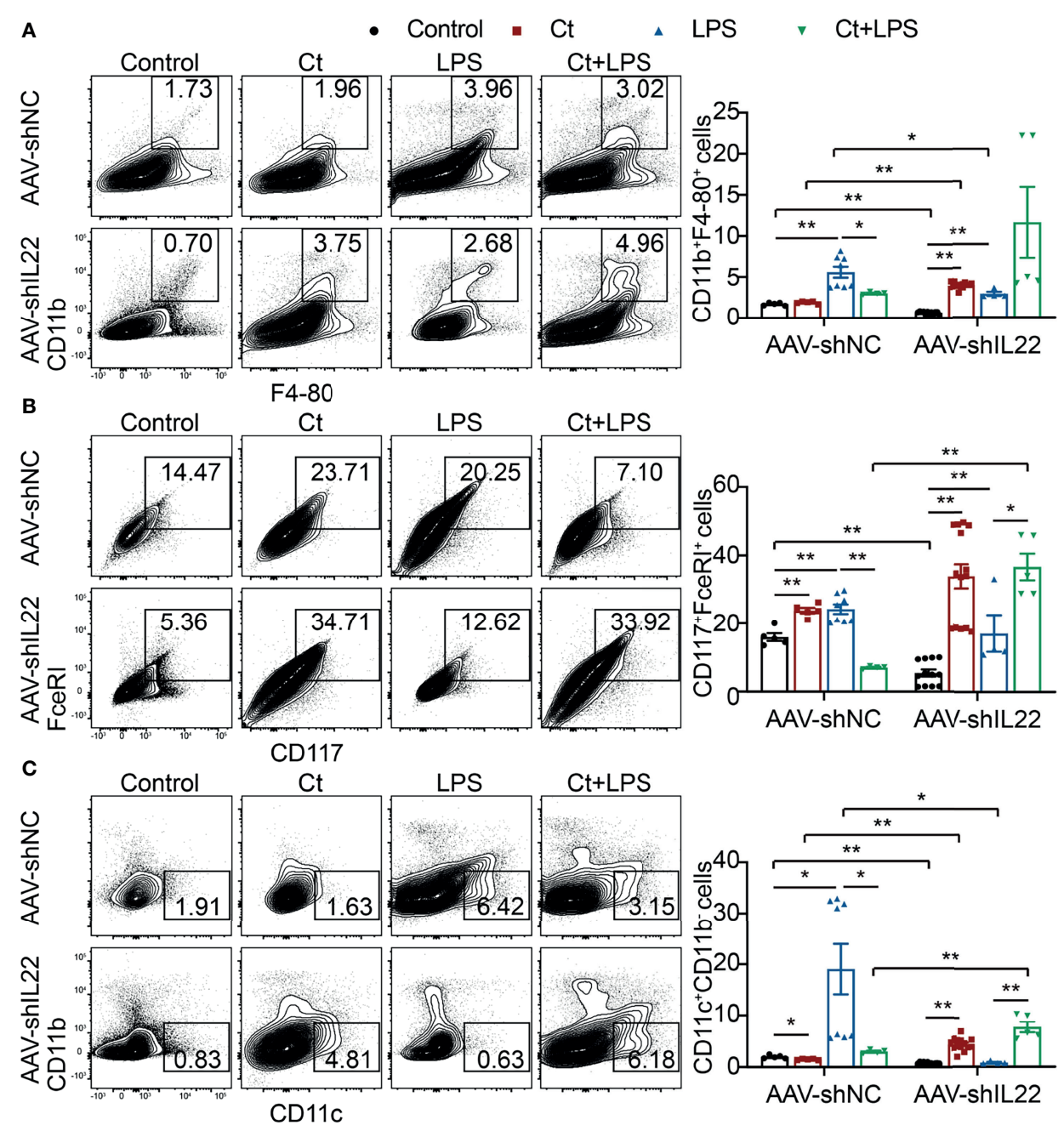

FIGURE 4 | Effects of Ct on the proportions of macrophages, mast cells, and DCs in the ileum. Flow cytometric analyses were conducted on 8-colour FACS Verse and three panels were used to analyzing the intestinal immune cells. The single cells were first gated on SSC-A vs FSC-A and FSC-A vs FSC-H, and then

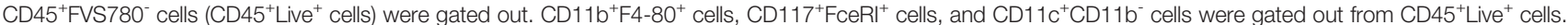
(A) Proportions of macrophages (CD11b ${ }^{+} F 4-80^{+}$cells). (B) Proportions of mast cells (CD117 ${ }^{+}$FceRI ${ }^{+}$cells). (C) Proportions of DCs (CD11 ${ }^{+}$CD11b cells). Each point presented a mouse. Data were presented as mean \pm SEM. The significant difference was analyzed by two-way unpaired $\mathrm{t}$-tests. ${ }^{\star} P<0.05,{ }^{\star \star} P<0.01$.

expression in mice treated AAV-shIL22 in response to LPS (Figure 3F). Although the structure of TJs was observed, the microvilli was damaged in the AAV-shIL22-Ct + LPS group (Figure 3G). Besides, after knocking down IL-22, Ct enhanced the mRNA expression of IL- 6 and had no effects on IL- $1 \beta$ and IL-10 in response to LPS (Figure 3H). Collectively, these findings indicated that $\mathrm{Ct}$ protected against LPS-induced dysfunction depending on IL-22 in the ileum.

The IL-22 receptor (IL-22R), composed of two heterodimeric subunits including IL-22RA1 and IL-10R2, is one of the IL-10 family of receptors (38). According to binding studies, IL-22 initially binds to IL-22RA1 since IL-22 has a high affinity for IL22RA1, and as such, the formation of the IL-22-IL-22RA1 complex enables secondary binding of the IL-10R2 subunit to activate downstream signaling (39). In this study, after knocking down IL-22, although Ct enhanced the mRNA expression of IL22 in response to LPS, no differences in the mRNA expression of IL-22RA1 in the ileum were observed between the LPS and Ct + LPS groups. IL-22BP, a soluble form of the IL-22RA1 subunit with a higher affinity to IL-22 than the membrane-bound IL22RA1 form, binds to IL-22 at an overlapping site to IL-22RA1 $(40,41)$. Compared with LPS group, Ct had no effects on mRNA expression of IL-22BP in response to LPS (Figure 3I).

\section{Ct Stimulates Th17 Cells to Produce IL-22 in the Lamina Propria}

IL-22, identified as one of the IL-10 members, is generally produced by a series of immune cells such as Th17 cells, ILC cells, and dendritic cells (DCs) $(42,43)$. According to the transcriptomic analyses, we identified some differentially 
expressed genes enriched in the cluster of differentiation (Supplemental Figure 3E), some of which have been defined as the surface biomarkers of immune cells, indicating that $\mathrm{Ct}$ might specifically regulate the function of intestinal immune cells. Our previous study also points out that Ct mainly targets Th17 cells in the ileum and supposes that Ct may stimulate Th17 cells to produce IL-22 in the ileum (14).

To investigate whether Ct regulates ileal immune cells in the in vivo interference feeding experiment, we isolated ileal lamina propria cells from AAV-shNC- and AAV-shIL22-treated mice to analyze the proportions of immune cells including macrophages, mast cells, DCs, ILC3s, CD4 ${ }^{+} \mathrm{T}$ cells, and $\mathrm{T}$ cell subsets in the ileum. As shown in Figure 4 and Supplemental Table 10, in the innate immune system, In mice treated with AAV-shNC, compared with LPS group, Ct decreased the proportions of macrophages, mast cells, and DCs in response to LPS in the ileum, which were consistent with our previous study (14). After knocking down IL-22, Ct enhanced the frequencies of mast cells and DCs in response to LPS. Numerous studies have revealed that IL-22 is induced by immune cells such as Th17 cells and ILC3s, thereby repairing epithelial damage (38, 44-48). Herein, as shown in Figure 5A, Supplemental Tables 11, 12 and Supplemental Figure 5, in mice treated with AAV-shNC, in response to LPS, Ct increased the proportions of Th1 cells, Tregs, and Th17 cells, while after knocking down IL-22, Ct decreased the numbers of Th1 cells and Tregs and had no effects on Th17 cells number. Besides, compared with LPS, Ct had no effects on the proportions of ILC3s in response to LPS (Figure 5B), indicating that $\mathrm{Ct}$ triggered Th17 cells rather than ILC3s in the ileum.

To better understand the origin and position of IL-22 production in the intestine, the ileal slices were stained with EpCAM, CD45, IL-22, and IL-22RA1 (Figure 6A). We first analyzed the colocalization of EpCAM, CD45, and IL-22 in the ileum (Figures 6B, C). IL- $22^{+} \mathrm{EpCAM}^{+} \mathrm{CD} 45^{+}$cells were observed in the intraepithelial lymphocyte and crypt, especially the crypt where IL-22 is highly expressed to promote the proliferation of intestinal stem cells $(29,49)$. According to the colocalization analysis of EpCAM and IL-22 using Pearson's R value. In response to LPS, Ct enhanced the colocalization of EpCAM and IL-22, suggesting that the IL-22 colocalized with EpCAM might be from the intraepithelial lymphocytes. Moreover, IL- $22^{+} \mathrm{CD} 45^{+}$cells were observed in the lamina propria after $\mathrm{Ct}$ treatment and $\mathrm{Ct}$ enhanced the whole number of $\mathrm{CD} 45^{+} \mathrm{IL}-22^{+}$cells in response to LPS. Ligation of the IL-22IL-22RA1-IL-10R2 complex is an essential step to mediate the biological effects of IL-22, thereby activating the downstream signaling pathways like the JAK-STAT pathway in epithelial cells and inducing renewal of epithelial cells $(38,50,51)$. In the above process, the IL-22RA1 determines the cellular sensitivity to IL-22
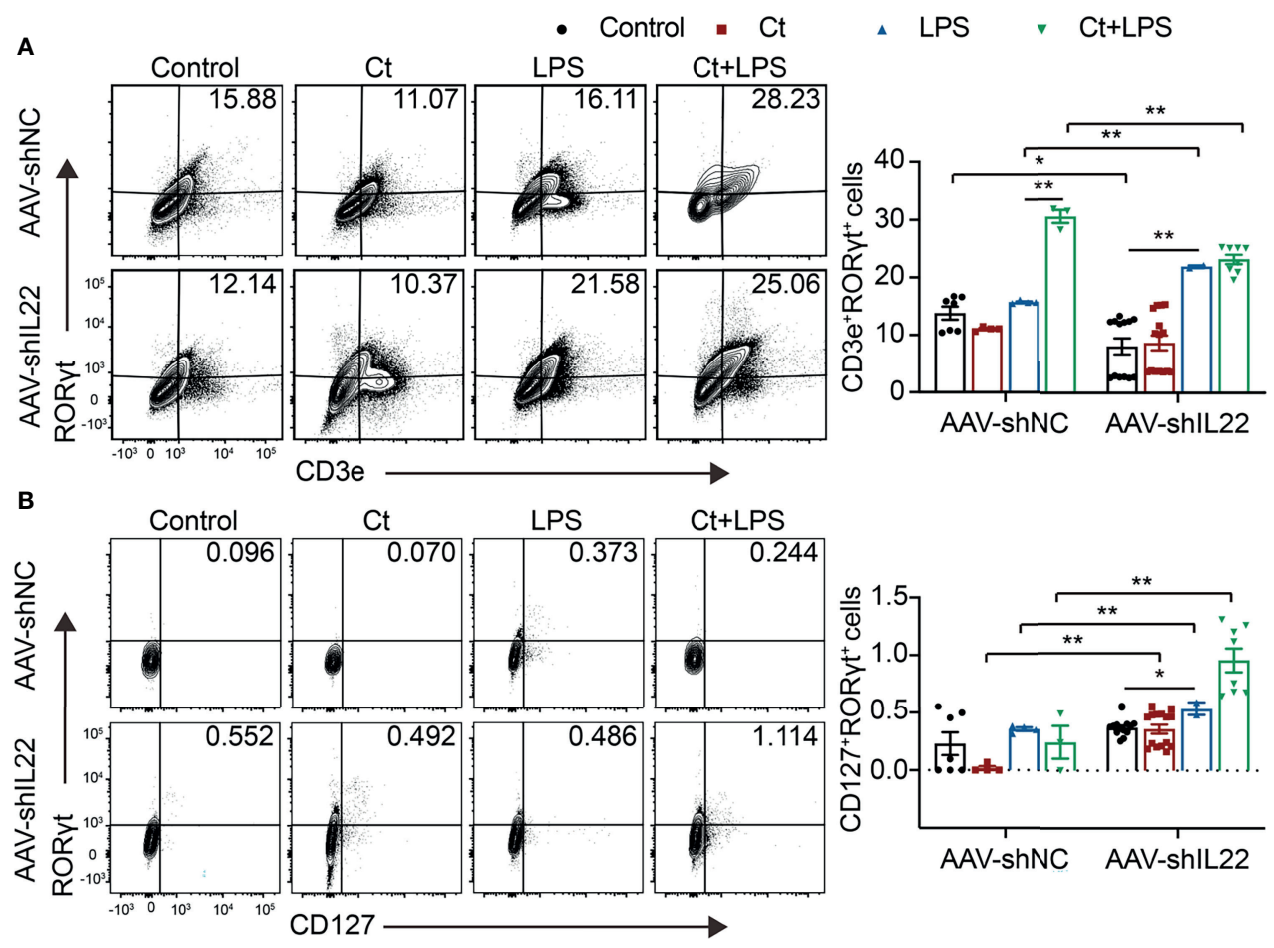

FIGURE 5 Ct stimulates Th17 cells rather than ILC3s in the ileum. Flow cytometric analyses were conducted on 8-colour FACS Verse and three panels were used to analyzing the intestinal immune cells. The single cells were first gated on SSC-A vs FSC-A and FSC-A vs FSC-H, and then CD45 ${ }^{+} \mathrm{FVS780}{ }^{-}$cells (CD45 ${ }^{+} \mathrm{Live}^{+}$cells) were gated out. CD3e $\mathrm{e}^{+} \mathrm{ROR} \gamma \mathrm{t}^{+}$cells were gated out from CD45 ${ }^{+} \mathrm{CD} 3 \mathrm{e}^{-}$cells. CD45 ${ }^{+} \mathrm{CD} 3 \mathrm{e}^{-}$cells were gated out from $\mathrm{CD} 45^{+} \mathrm{FVS} 780^{-}$cells and $\mathrm{CD} 127^{+} \mathrm{ROR} \gamma \mathrm{t}^{+}$cells were gated out from CD45 ${ }^{+} \mathrm{CD} 3 \mathrm{e}^{-}$cells. (A) Proportions of Th17 cells (CD45 ${ }^{+} \mathrm{CD} 3 \mathrm{e}^{+} \mathrm{ROR} \gamma \mathrm{t}^{+}$cells). (B) Proportions of ILC3s $\left(\mathrm{CD} 45^{+} \mathrm{CD} 3 \mathrm{e}^{-} \mathrm{CD} 127^{+} \mathrm{ROR}\right.$ ( $\left.\mathrm{t}^{+}\right)$. Each point presented a mouse. Data were presented as mean \pm SEM. The significant difference was analyzed by two-way unpaired t-tests. ${ }^{\star} P<0.05,{ }^{\star \star} P<0.01$. 

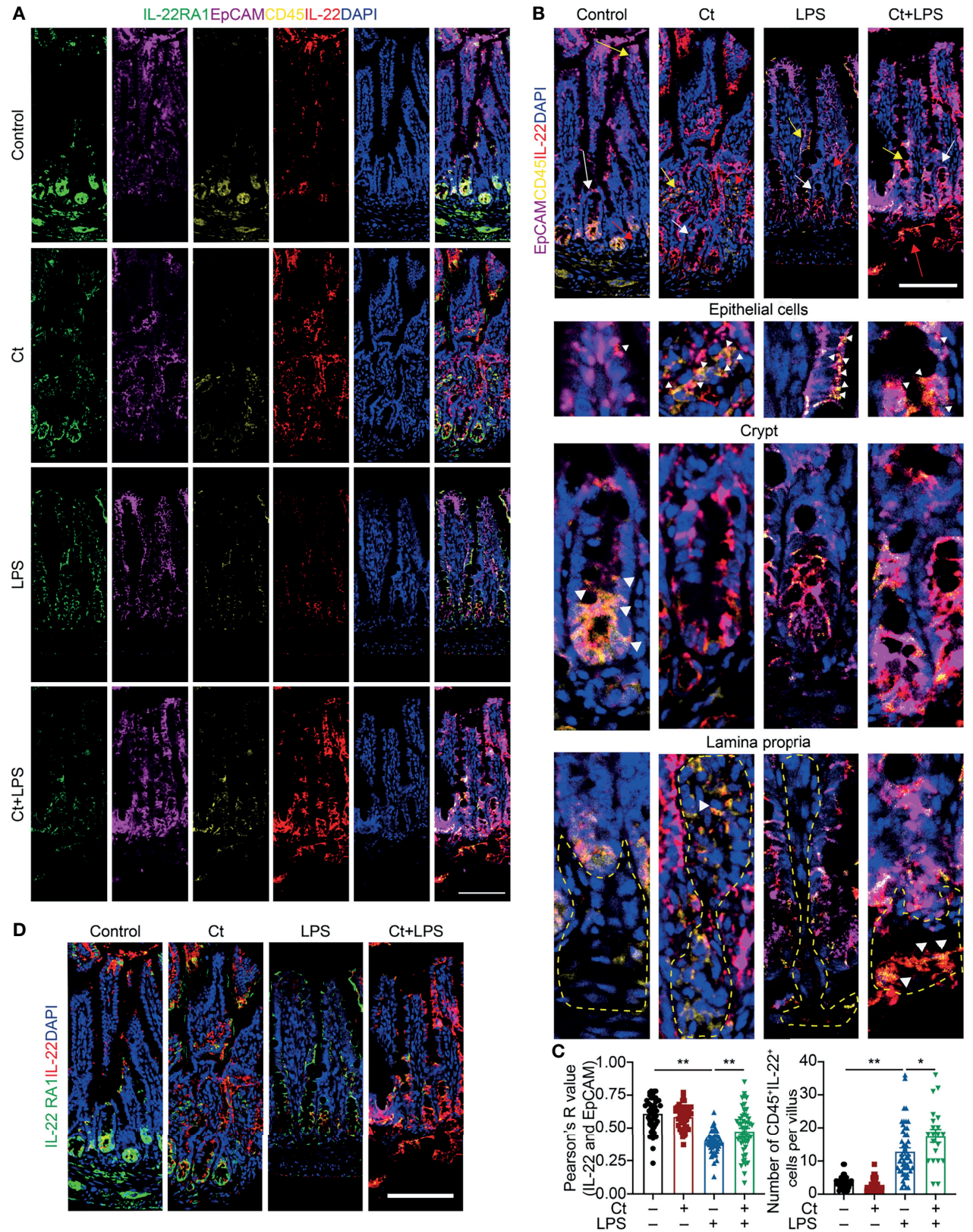

FIGURE 6 | Ct triggers immune cells to enhance the production of IL-22 in the ileal lamina propria. lleal slices were stained with IL-22 (red), IL-22RA1 (green), EpCAM (violet), CD45 (yellow), and DAPI (blue). (A) In situ hybridization of IL-22, IL-22RA1, EpCAM, and CD45. (B) Colocalization of EpCAM, CD45, and IL-22 in the epithelial position, crypt, and lamina propria, respectively. White triangle: localization, yellow arrow: epithelial cells, white arrow: crypt, red arrow: lamina propria (C) Analysis of IL-22 ${ }^{+} \mathrm{EpCAM}^{+}$and CD45 ${ }^{+} \mathrm{IL}-22^{+}$cells. (D) Colocalization of IL-22 and IL-22RA1. Scale bar: $100 \mu \mathrm{m}$. Data were presented as mean \pm SEM. The significant difference was analyzed by two-way unpaired t-tests. ${ }^{\star} P<0.05,{ }^{\star \star} P<0.01$.

and is mainly expressed in outer body barriers of respiratory like lungs, the gastrointestinal like stomach and intestine, the liver, the kidney, but not expressed in the bone marrow, spleen, or thymus containing high proportions of immune cells $(52,53)$. Herein, we observed that $\mathrm{Ct}$ induced higher colocalization of IL22 and IL-22RA1 in the epithelial cells (Figure 6D). Altogether, Ct stimulated the intestinal immune cells to produce IL-22 and triggered the combination of IL-22 with IL-22RA1. The ileal slices were then stained with ROR $\mathrm{t}$, EpCAM, CD3e, and IL-22 to determine whether IL-22 was produced by Th17 cells (Figure 7). In response to LPS, Ct enhanced the number of $\mathrm{ROR} \gamma \mathrm{t}^{+} \mathrm{CD} 3 \mathrm{e}^{+} \mathrm{IL}-22^{+}$cells. These results further suggested that Ct enhanced the level of IL-22 induced by Th17 cells in the lamina propria. 


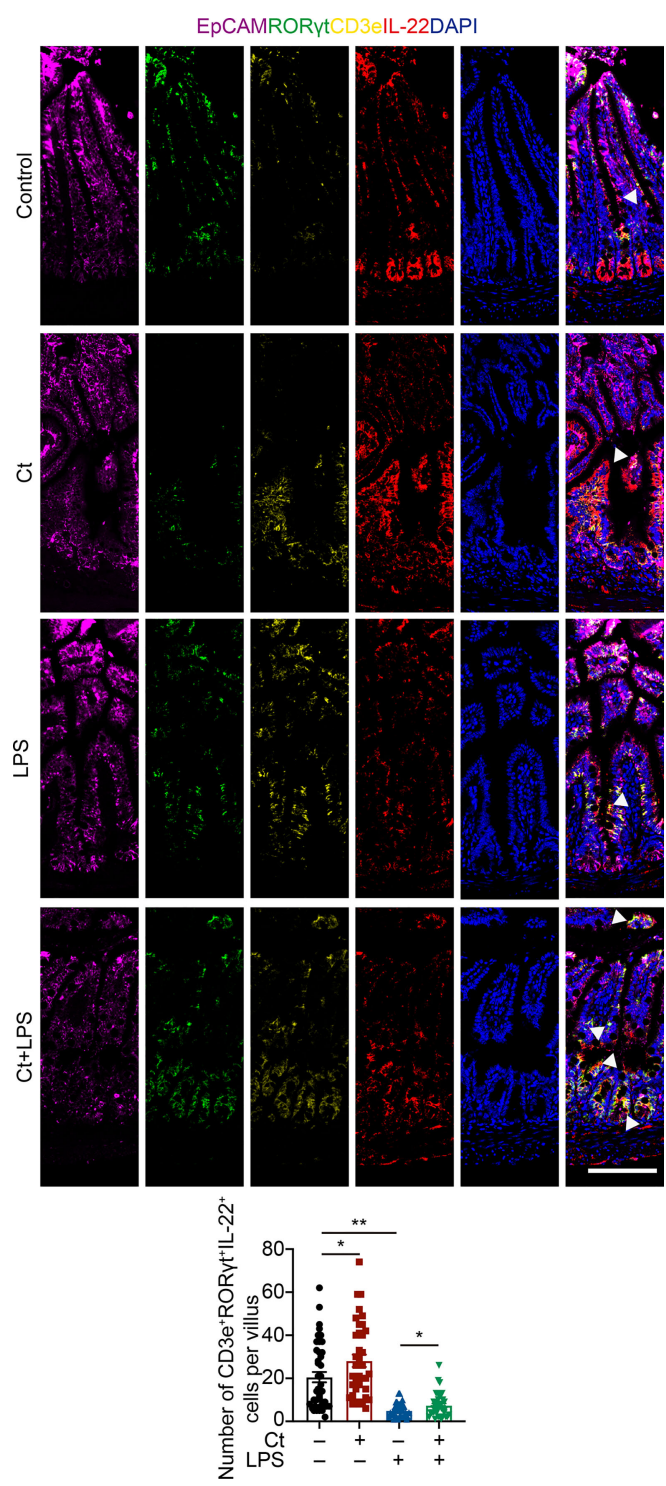

FIGURE 7 | Ct stimulates intestinal Th17 cells to enhance the production of IL-22 in the ileum. lleal slices were stained with EpCAM (violet), RORyt (green), CD3e (yellow), IL-22 (red), and DAPI (blue). The samples were from the continuous slices in Fig. 6 . White triangle: localization. Scale bar: $100 \mu$ m. Data were presented as mean \pm SEM. The significant difference was analyzed by two-way unpaired t-tests. ${ }^{\star} P<0.05,{ }^{\star \star} P<0.01$.

\section{The Role of Ct in Regulating Intestinal Health Was Independent of SCFAs}

Most probiotics regulate intestinal health via their metabolites like short-chain fatty acids (SCFAs) in the gut. Bacterial metabolites including short-chain acids (SCFAs), bile acids (BAs), and tryptophan metabolites, bacterial components, and bacteria themselves have been shown to play vital roles in maintaining epithelial integrity and modulating immune responses (54-56). Butyrate acid-producing Clostridia like Clostridium butyrium has been well studied in regulating intestinal health and inhibiting intestinal tumor development in both patients with IBD and animals with the mechanism of inhibiting histone deacetylase (HDAC) via SCFAs $(23,57)$.
Herein, we found that Ct dramatically decreased the levels of SCFAs in the intestine in response to LPS and the decreasing of SCFAs by Ct was independent on IL-22 (Figure 8), indicating that $\mathrm{Ct}$ protected against intestinal injury in a butyrate acidindependent manner.

\section{DISCUSSION}

Probiotics have been widely used in both humans and production animals. However, some inevitable problems must be considered for probiotics, mainly including the safety, the efficiency in treating intestinal diseases, the colonization in the gut, the 


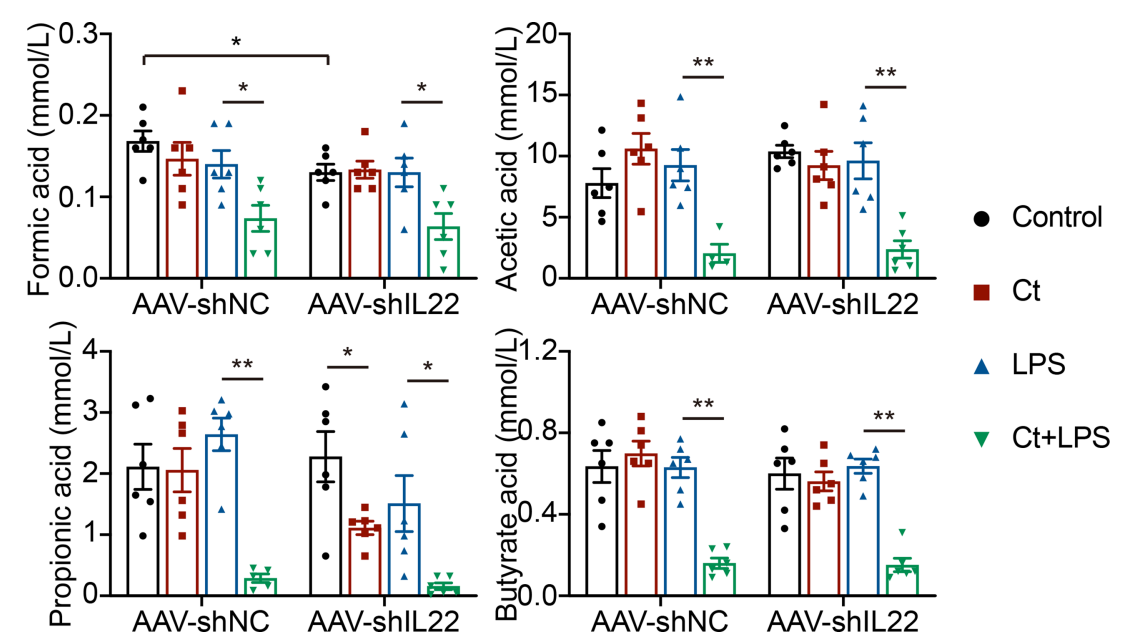

FIGURE 8 | Ct decreases the ileal levels of SCFAs in response to LPS. Data were presented as mean \pm SEM ( $\mathrm{n}=6)$. The significant difference was analyzed by twoway unpaired t-tests. ${ }^{\star} P<0.05,{ }^{\star \star} P<0.01$.

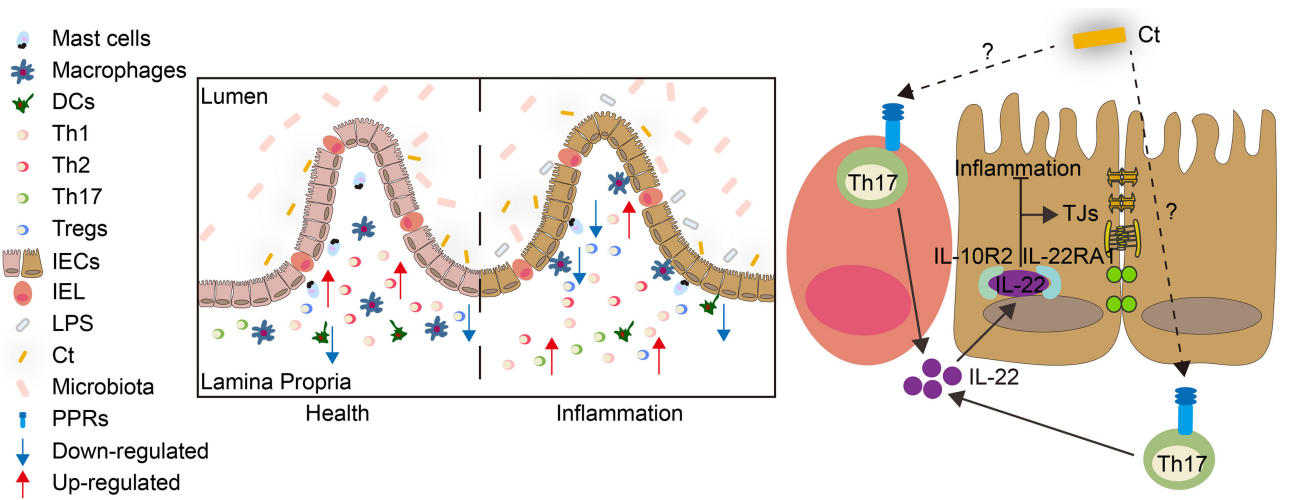

FIGURE 9 | Model for the function and mechanism of Ct in regulating intestinal health. Ct inhibited LPS-induced intestinal inflammation in the ileum. The protective role of Ct in the ileum presented an IL-22-dependent manner in vivo. In response to LPS, Ct enhanced the proportions of Th17 cells to produce IL-22, which combined with IL-22RA1 expressed in the epithelial cells, thereby maintaining the barrier function and alleviating inflammation in the ileum.

viability after passing through the gastrointestinal tract, and the effects on commensal microbiota (58). As for C. tyrobutyricum, our previous study has shown that $10^{8} \mathrm{CFU} / \mathrm{mL}$ C. tyrobutyricum could effectively alleviate LPS-induced inflammation in both the ileum and colon $(13,14)$. Moreover, the viability of C. tyrobutyricum reaches $90.03 \%$ after treatment in the simulated gastric fluid in vitro $(\mathrm{pH} 1.2)$ for $2 \mathrm{~h}$ and its viability reaches $62.12 \%$ after continuous treatment in the simulated intestinal fluid ( $\mathrm{pH}$ 7.4) for $4 \mathrm{~h}$ (59), suggesting that $C$. tyrobutyricum has considerable high gastrointestinal tolerance. Colonization is an essential characteristic of probiotics. Two decades-long debate views on colonization are existed, namely the colonization of the mucosa during supplementation and the post-supplementation persistence in the gut, which are limited by the abundance of probiotics in the assessment methods directly reflecting their colonization in the gut (58). Herein, we noticed that $C$. tyrobutyricum had the colonization capacity in the small intestine, while the accurate quantitative of viability was not fully revealed because of its weak colonization in the gut. Considering that $C$. tyrobutyricum effectively alleviated intestinal inflammation and prevented LPS-induced diarrhea, how to enhance the colonization or adhesion of C. tyrobutyricum on the gut mucosa is an imperative problem in the IBD therapy and the application in preventing diarrhea. A recent study revealed that the bacteria-derived biofilm contributes to the defense of bacteria under extreme conditions, such as physical forces and environmental attacks. The biofilm-coated probiotics present improved gastrointestinal tract tolerance and mucosal adhesion in animals and exhibit higher oral bioavailability and colonization than uncoated bacteria in the intestine (60), providing a feasible method to enhance the colonization or adhesion of C. tyrobutyricum in vivo. 
According to the transcriptomic analysis, compared with the Control, C. tyrobutyricum treatment alone decreased the expression of Cav1, Tacr2, and Adrb3, which were enriched in the process of neuro regulation and calcium signaling pathway according to the KEGG analysis. Moreover, we noticed that mice presented slowly in action during the gavage of C. tyrobutyricum. Whether C. tyrobutyricum alleviating intestinal dysfunction via the brain-gut axis or intestinal nervous regulation needs further study. Considering that LPS induced the metabolic disorder and C. tyrobutyricum reversed the metabolic processes like fatty acids synthesis and degradation in response to LPS, whether $C$. tyrobutyricum orchestrating the immune response and metabolism needs to be revealed in the future study.

IL-22, discovered by Gurney's group and Renauld's group in $2000(61,62)$, is highly expressed in some gastrointestinal diseases like IBD (27, 63-65). However, IL-22 repairs tissue damage and prevents pathogens via promoting the regeneration of epithelial cells and the production of antimicrobial molecules when it is under proper control $(38,45,46)$. The fuzzy boundary of IL-22 between inhibitor and inducer for colitis may present a dose-/time-dependent manner in the intestine. In this study, we found that C. tyrobutyricum alleviated intestinal inflammation depending on IL-22 produced by Th17 cells. Strangely, LPS stimulation decreased the mRNA expression of IL- 6 and proportions of immune cells like macrophages and DCs in the ileum after knocking down IL-22. Some similar results were also observed in the colon (13). Unlike the earlier study in which IL22 level in the intestine is enhanced in response to LPS (64), herein, the expression of IL-22 in the ileum was decreased in response to LPS. Thus, whether LPS-induced intestinal inflammation depending on IL-22 needs further investigation. More importantly, to fully understand the role of $C$. tyrobutyricum in the intestine, chemical methods like DSS should be considered to induce IBD in our future research.

IECs receive signals from nutrients, commensal microbes, or pathogens, orchestrating the communication between the commensal microorganisms and immune cells under physiological and inflammatory conditions. These signals are classified into three types, namely the microbiota, the microbial components, and the microbial metabolites (2). For most studies, probiotics regulate intestinal health via their metabolites like SCFAs, bile acids, and tryptophan metabolites, which have been revealed to maintain the interactions among microbiota, epithelial cells, and immune cells in the gut. The SCFAs activate the G protein-coupled receptors (GPRs) expressed in the epithelial cells to inhibit inflammation via producing cytokines like IL-18 (66), concerting the communication between microbiota and IECs. The SCFAs can also regulate the interactions between microbiota and immune cells with the mechanism of stimulating the IL-10 producing Foxp $3^{+}$Tregs via inhibiting HDAC, thereby alleviating colitis $(56,66)$. As one of Clostridium strains producing high levels of butyrate acid in vitro, C. tyrobutyricum was unable to enhance the levels of SCFAs in the ileum in response to LPS. In this study, we suggested that $C$. tyrobutyricum might play a protective role in the intestine via stimulating the metabolites except for SCFAs. Further investigation in whether
C. tyrobutyricum could trigger the production of metabolites contributing to the stimulation of Th17 cells to produce IL-22 in the gut based on metabolome should be considered.

The present study revealed that $C$. tyrobutyricum alleviated LPSinduced intestinal inflammation and prevented diarrhea in mice with the mechanism of triggering Th17 cells in the lamina propria to induce IL-22 production, which combined with IL-22RA1 expressed in the epithelial cells (Figure 9). The results indicated that $C$. tyrobutyricum could be a potential probiotic in regulating intestinal health and provided a scientific basis for its application in preventing diarrhea and inflammation in the ileum.

\section{DATA AVAILABILITY STATEMENT}

The original contributions presented in the study are publicly available. This data can be found here: https://www.ncbi.nlm.nih. gov/sra/?term=PRJNA756726.

\section{ETHICS STATEMENT}

The animal study was reviewed and approved by The Institutional Animal Care and Use Committee of Zhejiang University (Approval numbers: ZJU20200005, ZJU20200040).

\section{AUTHOR CONTRIBUTIONS}

MW took charge of the project administration. ZX designed the whole experiment. ZX and LL performed all in vivo and in vitro experiments. ZX and LL analyzed and checked the data. ZX wrote the original manuscript. LL revised the manuscript. XP and WS performed the bacterial colonization experiment and cultured the bacteria. YJ performed flow cytometry experiments. ZX, LL, XP, and WS collected the tissue samples. S-TY revised and copy-edited the paper. All authors contributed to the article and approved the submitted version.

\section{FUNDING}

This study was jointly funded by the National Key Research \& Development Program of China (No. 2018YFE0112700) and Key Research \& Development Program of Zhejiang Province (No. 2019C02005).

\section{ACKNOWLEDGMENTS}

We would like to thank all members from Laboratory Animal Center, Zhejiang University for taking care of all experimental mice. We show great thanks to all members from the Bioultrastructure analysis Lab. of Analysis Center of Agrobiology and Environmental Sciences, Zhejiang University for their supports in electron microscopy and confocal microscopy. 
We would like to thank Bojing Liu from Institute of Feed Science, Zhejiang University for her kind help in SCFAs measurement. We would also like to thank Weiren Dong, Junhong Wang, and Yuanyuan Zhang from lab platform of College of Animal Sciences, Zhejiang University for their technical supports.

\section{REFERENCES}

1. Viswanathan VK, Hodges K, Hecht G. Enteric Infection Meets Intestinal Function: How Bacterial Pathogens Cause Diarrhoea. Nat Rev Microbiol (2009) 7:110-9. doi: 10.1038/nrmicro2053

2. Kayama H, Okumura R, Takeda K. Interaction Between the Microbiota, Epithelia, and Immune Cells in the Intestine. Annu Rev Immunol (2020) 38:23-48. doi: 10.1146/annurev-immunol-070119-115104

3. Helene H, Jacqyekube C, Bergere J. A High-Molecular-Mass Cell Wall Protein Released From Clostridium Tyrobutyricum by Heat Treatment. FEBS Lett (1984) 174:284-8. doi: 10.1016/0014-5793(84)81174-6

4. Bergere JL, Commissaire H, Hayes J. Major Protein Components in the Cell Envelope of Clostridium Tyrobutyricum. Annu Inst pasteur Microbiol (1986) 127:271-82. doi: 10.1016/s0769-2609(86)80117-x

5. Xiao Z, Cheng C, Bao T, Liu L, Wang B, Tao W, et al. Production of Butyric Acid From Acid Hydrolysate of Corn Husk in Fermentation by Clostridium Tyrobutyricum: Kinetics and Process Economic Analysis. Biotechnol Biofuels (2018) 11:164. doi: 10.1186/s13068-018-1165-1

6. Fu H, Yu L, Lin M, Wang J, Xiu Z, Yang ST. Metabolic Engineering of Clostridium Tyrobutyricum for Enhanced Butyric Acid Production From Glucose and Xylose. Metab Eng (2017) 40:50-8. doi: 10.1016/j.ymben.2016.12.014

7. Zhang J, Yu L, Lin M, Yan Q, Yang ST. N-Butanol Production From Sucrose and Sugarcane Juice by Engineered Clostridium Tyrobutyricum Overexpressing Sucrose Catabolism Genes and Adhe2. Bioresour Technol (2017) 233:51-7. doi: 10.1016/j.biortech.2017.02.079

8. Hudcovic T, Kolinska J, Klepetar J, Stepankova R, Rezanka T, Srutkova D, et al. Protective Effect of Clostridium Tyrobutyricum in Acute Dextran Sodium Sulphate-Induced Colitis: Differential Regulation of Tumour Necrosis Factor-A and Interleukin-18 in BALB/C and Severe Combined Immunodeficiency Mice. Clin Exp Immunol (2012) 167:356-65. doi: $10.1111 / j .1365-2249.2011 .04498 . x$

9. Al-Asmakh M, Stukenborg JB, Reda A, Anuar F, Strand ML, Hedin L, et al. The Gut Microbiota and Developmental Programming of the Testis in Mice. PloS One (2014) 9:e103809. doi: 10.1371/journal.pone.0103809

10. Hu X, Guo J, Xu M, Jiang P, Yuan X, Zhao C, et al. Clostridium Tyrobutyricum Alleviates Staphylococcus Aureus-Induced Endometritis in Mice by Inhibiting Endometrial Barrier Disruption and Inflammatory Response. Food Funct (2019) 10:6699-710. doi: 10.1039/c9fo00654k

11. Liu T, Zhu L, Zhu Z, Jiang L. Genome Sequence Analysis of Clostridium Tyrobutyricum, a Promising Microbial Host for Human Health and Industrial Applications. Curr Microbiol (2020) 77:3685-94. doi: 10.1007/s00284-020-02175-0

12. Xiao Z, Liu L, Tao W, Pei X, Wang G, Wang M. Clostridium Tyrobutyricum Protect Intestinal Barrier Function From LPS-Induced Apoptosis via P38/JNK Signaling Pathway in IPEC-J2 Cells. Cell Physiol Biochem (2018) 46:1779-92. doi: $10.1159 / 000489364$

13. Xiao Z, Liu L, Jin Y, Pei X, Sun W, Wang M. Clostridium Tyrobutyricum Protects Against LPS-Induced Colonic Inflammation via IL-22 Signaling in Mice. Nutrients (2021) 13:215. doi: 10.3390/nu13010215

14. Xiao Z, Liu L, Jin Y, Pei X, Sun W, Wang M. A Potential Prophylactic Probiotic for Inflammatory Bowel Disease: The Overall Investigation of Clostridium Tyrobutyricum ATCC25755 Attenuates LPS-Induced Inflammation via Regulating Intestinal Immune Cells. Mol Nutr Food Res (2021) 65:e2001213. doi: $10.1002 / \mathrm{mnfr} .202001213$

15. Scott CL, Bain CC, Mowat AM. Isolation and Identification of Intestinal Myeloid Cells. Methods Mol Biol (2017) 1559:223-39. doi: 10.1007/978-14939-6786-5_15

16. Heli UH, Emma P, Petra N, William A. Isolation of Cells From Human Intestinal Tissue. Bio-Protocol (2014) 4:1-8. doi: 10.21769/BioProtoc.1092

17. Hoving LR, Heijink M, van Harmelen V, van Dijk KW, Giera M. Gc-Ms Analysis of Short-Chain Fatty Acids in Feces, Cecum Content, and Blood

\section{SUPPLEMENTARY MATERIAL}

The Supplementary Material for this article can be found online at: https://www.frontiersin.org/articles/10.3389/fimmu.2021.758227/ full\#supplementary-material

Samples. Methods Mol Biol (2018) 1730:247-56. doi: 10.1007/978-1-49397592-1_17

18. Niccolai E, Baldi S, Ricci F, Russo E, Nannini G, Menicatti M, et al. Evaluation and Comparison of Short Chain Fatty Acids Composition in Gut Diseases. World J Gastroenterol (2019) 25:5543-58. doi: 10.3748/wjg.v25.i36.5543

19. Swallow CJ, Partridge EA, Macmillan JC, Tajirian T, DiGuglielmo GM, Hay K, et al. Alpha2hs-Glycoprotein, an Antagonist of Transforming Growth Factor Beta In Vivo, Inhibits Intestinal Tumor Progression. Cancer Res (2004) 64:6402-9. doi: 10.1158/0008-5472.CAN-04-1117

20. Hu LH, Fan YJ, Li Q, Guan JM, Qu B, Pei FH, et al. Bortezomib Protects Against Dextran Sulfate Sodiuminduced Ulcerative Colitis in Mice. Mol Med Rep (2017) 15:4093-9. doi: 10.3892/mmr.2017.6524

21. Nowarski R, Gagliani N, Huber S, Flavell RA. Innate Immune Cells in Inflammation and Cancer. Cancer Immunol Res (2013) 1:77-84. doi: 10.1158/2326-6066.CIR-13-0081

22. Iwasaki A, Medzhitov R. Control of Adaptive Immunity by the Innate Immune System. Nat Immunol (2015) 16:343-53. doi: 10.1038/ni.3123

23. Hayashi A, Sato T, Kamada N, Mikami Y, Matsuoka K, Hisamatsu T, et al. A Single Strain of Clostridium Butyricum Induces Intestinal IL-10-Producing Macrophages to Suppress Acute Experimental Colitis in Mice. Cell Host Microbe (2013) 13:711-22. doi: 10.1016/j.chom.2013.05.013

24. Zhu S, Ding S, Wang P, Wei Z, Pan W, Palm NW, et al. Nlrp9b Inflammasome Restricts Rotavirus Infection in Intestinal Epithelial Cells. Nature (2017) 546:667-70. doi: 10.1038/nature22967

25. Chikano S, Sawada K, Shimoyama T, Kashiwamura S-I, Sugihara A, Sekikawa K, et al. Il-18 and Il-12 Induce Intestinal Inflammation and Fatty Liver in Mice in an IFN- $\gamma$ Dependent Manner. Gut (2000) 47:779-86. doi: 10.1136/gut.47.6.779

26. Sivakumar PV, Westrich GM, Kanaly S, Garka K, Born TL, Derry JMJ, et al. Interleukin 18 Is a Primary Mediator of the Inflammation Associated With Dextran Sulphate Sodium Induced Colitis: Blocking Interleukin 18 Attenuates Intestinal Damage. Gut (2002) 50:812-20. doi: 10.1136/gut.50.6.812

27. Brand S, Beigel F, Olszak T, Zitzmann K, Eichhorst ST, Otte JM, et al. Il-22 Is Increased in Active Crohn's Disease and Promotes Proinflammatory Gene Expression and Intestinal Epithelial Cell Migration. Am J Physiol Gastrointest Liver Physiol (2006) 290:G827-38. doi: 10.1152/ajpgi.00513.2005

28. Lee JS, Tato CM, Joyce-Shaikh B, Gulen MF, Cayatte C, Chen Y, et al. Interleukin-23-Independent Il-17 Production Regulates Intestinal Epithelial Permeability. Immunity (2015) 43:727-38. doi: 10.1016/j.immuni.2015.09.003

29. Hanash AM, Dudakov JA, Hua G, O'Connor MH, Young LF, Singer NV, et al. Interleukin-22 Protects Intestinal Stem Cells From Immune-Mediated Tissue Damage and Regulates Sensitivity to Graft Versus Host Disease. Immunity (2012) 37:339-50. doi: 10.1016/j.immuni.2012.05.028

30. Nowarski R, Jackson R, Gagliani N, de Zoete MR, Palm NW, Bailis W, et al. Epithelial IL-18 Equilibrium Controls Barrier Function in Colitis. Cell (2015) 163:1444-56. doi: 10.1016/j.cell.2015.10.072

31. Jakovcevski M, Bharadwaj R, Straubhaar J, Gao G, Gavin DP, Jakovcevski I, et al. Prefrontal Cortical Dysfunction After Overexpression of Histone Deacetylase 1. Biol Psychiatry (2013) 74:696-705. doi: 10.1016/j.biopsych.2013.03.020

32. Fagarasan S, Kawamoto S, Kanagawa O, Suzuki K. Adaptive Immune Regulation in the Gut: T Cell-Dependent and T Cell-Independent Iga Synthesis. Annu Rev Immunol (2010) 28:243-73. doi: 10.1146/annurevimmunol-030409-101314

33. Mowat AM. Anatomical Basis of Tolerance and Immunity to Intestinal Antigens. Nat Rev Immunol (2003) 3:331-41. doi: 10.1038/nri1057

34. Neefjes J, Jongsma ML, Paul P, Bakke O. Towards a Systems Understanding of MHC Class I and MHC Class II Antigen Presentation. Nat Rev Immunol (2011) 11:823-36. doi: 10.1038/nri3084

35. Unanue ER, Turk V, Neefjes J. Variations in MHC Class II Antigen Processing and Presentation in Health and Disease. Annu Rev Immunol (2016) 34:26597. doi: 10.1146/annurev-immunol-041015-055420 
36. Posovszky C, Sirin M, Jacobsen E, Lorenz M, Schwarz K, Schmidt-Choudhury A, et al. Persisting Enteropathy and Disturbed Adaptive Mucosal Immunity Due to MHC Class II Deficiency. Clin Immunol (2019) 203:125-33. doi: 10.1016/j.clim.2019.04.012

37. Meital GB, Christoph AT, Serafini N, Winter DR, Giladi A, Lara-Astiaso D, et al. The Spectrum and Regulatory Landscape of Intestinal Innate Lymphoid Cells Are Shaped by the Microbiome. Cell (2016) 166:1231-46.e13. doi: 10.1016/j.cell.2016.07.043

38. Dudakov JA, Hanash AM, van den Brink MR. Interleukin-22: Immunobiology and Pathology. Annu Rev Immunol (2015) 33:747-85. doi: 10.1146/annurev-immunol-032414-112123

39. Bleicher L, de Moura P, Watanabe L, Colau D, Dumoutier L, Renauld J, et al. Crystal Structure of the IL-22/IL-22r1 Complex and Its Implications for the IL-22 Signaling Mechanism. FEBS Lett (2008) 582:2985-92. doi: 10.1016/ j.febslet.2008.07.046

40. Jones BC, Logsdon NJ, Walter MR. Structure of IL-22 Bound to Its HighAffinity IL-22r1 Chain. Structure (2008) 16:1333-44. doi: 10.1016/ j.str.2008.06.005

41. Wu PW, Li J, Kodangattil SR, Luxenberg DP, Bennett F, Martino M, et al. IL22r, IL-10R2, and Il-22bp Binding Sites Are Topologically Juxtaposed on Adjacent and Overlapping Surfaces of IL-22. J Mol Biol (2008) 382:1168-83. doi: 10.1016/j.jmb.2008.07.046

42. Shabgah AG, Navashenaq JG, Shabgah OG, Mohammadi H, Sahebkar A. Interleukin-22 in Human Inflammatory Diseases and Viral Infections. Autoimmun Rev (2017) 16:1209-18. doi: 10.1016/j.autrev.2017.10.004

43. Ouyang W, Rutz S, Crellin NK, Valdez PA, Hymowitz SG. Regulation and Functions of the IL-10 Family of Cytokines in Inflammation and Disease. Annu Rev Immunol (2011) 29:71-109. doi: 10.1146/annurev-immunol031210-101312

44. Perez LG, Kempski J, McGee HM, Pelzcar P, Agalioti T, Giannou A, et al. TGF-Beta Signaling in Th17 Cells Promotes IL-22 Production and ColitisAssociated Colon Cancer. Nat Commun (2020) 11:2608. doi: 10.1038/s41467020-16363-w

45. Wolk K, Witte E, Wallace E, Docke WD, Kunz S, Asadullah K, et al. IL-22 Regulates the Expression of Genes Responsible for Antimicrobial Defense, Cellular Differentiation, and Mobility in Keratinocytes: A Potential Role in Psoriasis. Eur J Immunol (2006) 36:1309-23. doi: 10.1002/eji.200535503

46. Liang SC, Tan XY, Luxenberg DP, Karim R, Kyriaki DJ, Collins M, et al. Interleukin (IL)-22 and IL-17 Are Coexpressed by Th17 Cells and Cooperatively Enhance Expression of Antimicrobial Peptides. J Exp Med (2006) 203:2271-9. doi: 10.1084/jem.20061308

47. Zelante T, Iannitti RG, Cunha C, De Luca A, Giovannini G , Pieraccini G, et al. Tryptophan Catabolites From Microbiota Engage Aryl Hydrocarbon Receptor and Balance Mucosal Reactivity via Interleukin-22. Immunity (2013) 39:372-85. doi: 10.1016/j.immuni.2013.08.003

48. Yang W, Yu T, Huang X, Bilotta AJ, Xu L, Lu Y, et al. Intestinal MicrobiotaDerived Short-Chain Fatty Acids Regulation of Immune Cell IL-22 Production and Gut Immunity. Nat Commun (2020) 11:4457. doi: 10.1038/ s41467-020-18262-6

49. Diefenbach A. Interleukin-22, the Guardian of the Intestinal Stem Cell Niche? Immunity (2012) 37:196-8. doi: 10.1016/j.immuni.2012.08.007

50. Hou Q, Ye L, Liu H, Huang L, Yang Q, Turner JR, et al. Lactobacillus Accelerates Iscs Regeneration to Protect the Integrity of Intestinal Mucosa Through Activation of STAT3 Signaling Pathway Induced by LPLs Secretion of IL-22. Cell Death Differ (2018) 25:1657-70. doi: 10.1038/s41418-018-0070-2

51. Aden K, Tran F, Ito G, Sheibani-Tezerji R, Lipinski S, Kuiper JW, et al. Atg16L1 Orchestrates Interleukin-22 Signaling in the Intestinal Epithelium via cGAS-STING. J Exp Med (2018) 215:2868-86. doi: 10.1084/jem.20171029

52. Witte E, Witte K, Warszawska K, Sabat R, Wolk K. Interleukin-22: A Cytokine Produced by T, NK and NKT Cell Subsets, With Importance in the Innate Immune Defense and Tissue Protection. Cytokine Growth Factor Rev (2010) 21:365-79. doi: 10.1016/j.cytogfr.2010.08.002

53. Wolk K, Kunz S, Witte E, Friedrich M, Asadullah K, Sabat R. Il-22 Increases the Innate Immunity of Tissues. Immunity (2004) 21:241-54. doi: 10.1016/ j.immuni.2004.07.007
54. Furusawa Y, Obata Y, Fukuda S, Endo TA, Nakato G, Takahashi D, et al. Commensal Microbe-Derived Butyrate Induces the Differentiation of Colonic Regulatory T Cells. Nature (2013) 504:446-50. doi: 10.1038/nature12721

55. Park J, Kim M, Kang SG, Jannasch AH, Cooper B, Patterson J, et al. ShortChain Fatty Acids Induce Both Effector and Regulatory T Cells by Suppression of Histone Deacetylases and Regulation of the Mtor-S6k Pathway. Mucosal Immunol (2015) 8:80-93. doi: 10.1038/mi.2014.44

56. Smith PM, Howitt MR, Panikov N, Michaud M, Gallini CA, Bohlooly YM, et al. The Microbial Metabolites, Short-Chain Fatty Acids, Regulate Colonic Treg Cell Homeostasis. Science (2013) 341:569-73. doi: 10.1126/ science. 1241165

57. Chen DF, Jin DC, Huang SM, Wu JY, Xu MQ, Liu TY, et al. Clostridium Butyricum, a Butyrate-Producing Probiotic, Inhibits Intestinal Tumor Development Through Modulating Wnt Signaling and Gut Microbiota. Cancer Lett (2020) 469:456-67. doi: 10.1016/j.canlet.2019.11.019

58. Suez J, Zmora N, Segal E, Elinav E. The Pros, Cons, and Many Unknowns of Probiotics. Nat Med (2019) 25:716-29. doi: 10.1038/s41591-019-0439-x

59. Yaqoob MU, Wang B, Pei X, Xiao Z, Sun W, Jin Y, et al. Microencapsulation of Clostridium Tyrobutyricum by Spray Drying Method and Its Characteristics in-Vitro. Pakistan Veterinary J (2020) 40:419-24. doi: 10.29261/pakvetj/2020.046

60. Wang X, Cao Z, Zhang M, Meng L, Ming Z, Liu J. Bioinspired Oral Delivery of Gut Microbiota by Self-Coating With Biofilms. Sci Adv (2020) 6:eabb1952. doi: 10.1126/sciadv.abb1952.eCollection2020Jun

61. Xie MH, Aggarwal S, Ho WH, Foster J, Zhang Z, Stinson J, et al. Interleukin (IL)-22, a Novel Human Cytokine That Signals Through the Interferon Receptor-Related Proteins Crf2-4 and IL-22r. J Biol Chem (2000) 275:31335-9. doi: 10.1074/jbc.M005304200

62. Dumoutier L, Roost L, Ameye G, Michaux L, Renauld J. IL-TIF/IL-22: Genomic Organization and Mapping of the Human and Mouse Genes. Genes Immun (2000) 1:488-94. doi: 10.1038/sj.gene.6363716

63. Kamanaka M, Huber S, Zenewicz LA, Gagliani N, Rathinam C, Connor WOJr., et al. Memory/Effector (Cd45rb(Lo)) CD4 T Cells Are Controlled Directly by IL-10 and Cause IL-22-Dependent Intestinal Pathology. J Exp Med (2011) 208:1027-40. doi: 10.1084/jem.20102149

64. Wolk K, Witte E, Hoffmann U, Doecke WD, Endesfelder S, Asadullah K, et al. IL-22 Induces Lipopolysaccharide-Binding Protein in Hepatocytes: A Potential Systemic Role of IL-22 in Crohn's Disease. J Immunol (2007) 178:5973-81. doi: 10.4049/jimmunol.178.9.5973

65. Mortha A, Chudnovskiy A, Hashimoto D, Bogunovic M, Spencer SP, Belkaid $\mathrm{Y}$, et al. Microbiota-Dependent Crosstalk Between Macrophages and Ilc3 Promotes Intestinal Homeostasis. Science (2014) 343:1249288. doi: 10.1126/ science. 1249288

66. Singh N, Gurav A, Sivaprakasam S, Brady E, Padia R, Shi H, et al. Activation of GPR109a, Receptor for Niacin and the Commensal Metabolite Butyrate, Suppresses Colonic Inflammation and Carcinogenesis. Immunity (2014) 40:128-39. doi: 10.1016/j.immuni.2013.12.007

Conflict of Interest: The authors declare that the research was conducted in the absence of any commercial or financial relationships that could be construed as a potential conflict of interest.

Publisher's Note: All claims expressed in this article are solely those of the authors and do not necessarily represent those of their affiliated organizations, or those of the publisher, the editors and the reviewers. Any product that may be evaluated in this article, or claim that may be made by its manufacturer, is not guaranteed or endorsed by the publisher.

Copyright $\odot 2021$ Xiao, Liu, Pei, Sun, Jin, Yang and Wang. This is an open-access article distributed under the terms of the Creative Commons Attribution License (CC BY). The use, distribution or reproduction in other forums is permitted, provided the original author(s) and the copyright owner(s) are credited and that the original publication in this journal is cited, in accordance with accepted academic practice. No use, distribution or reproduction is permitted which does not comply with these terms. 